\title{
The HELLAS2XMM survey
}

\section{Spectroscopic identification of super-EROs hosting AGNs ${ }^{\star}$}

\author{
R. Maiolino ${ }^{1}$, M. Mignoli ${ }^{2}$, L. Pozzetti ${ }^{2}$, P. Severgnini ${ }^{3}$, M. Brusa ${ }^{4}$, C. Vignali ${ }^{5}$, S. Puccetti ${ }^{6}$, P. Ciliegi $^{2}$, \\ F. Cocchia ${ }^{6}$, A. Comastri ${ }^{2}$, F. Fiore ${ }^{6}$, F. La Franca ${ }^{7}$, G. Matt ${ }^{7}$, S. Molendi ${ }^{8}$, and G. C. Perola ${ }^{7}$
}

1 INAF - Osservatorio Astrofisico di Arcetri, L.go E. Fermi 5, 50125 Firenze, Italy e-mail: maiolino@arcetri.astro.it

2 INAF - Osservatorio Astronomico di Bologna, via Ranzani 1, 40127 Bologna, Italy e-mail: [marco.mignoli; lucia.pozzetti; andrea.comastri]@bo.astro.it

3 INAF - Osservatorio Astronomico di Brera 28, 20121 Milano, Italy e-mail: paola@brera.mi .astro.it

${ }^{4}$ Max-Planck-Institut für Extraterrestrische Physik, Garching, Germany e-mail: marcella@mpe.mpg.de

5 Dipartimento di Astronomia, Universitá di Bologna, via Ranzani 1, 40127 Bologna, Italy e-mail: cristian.vignali@bo.astro.it

6 INAF - Osservatorio Astronomico di Roma, via Frascati 33, 00040 Monteporzio, Italy e-mail: [cocchia; fiore; puccetti] @mporzio.astro.it

7 Dipartimento di Fisica Universitá di Roma Tre, via della Vasca Navale 84, 00146 Roma, Italy e-mail: [lafranca;matt; perola]@fis.uniroma3.it

8 IASF-CNR, Istituto di Fisica Cosmica, via Bassini 15, 20133 Milano, Italy e-mail: silvano@mi.iasf.cnr.it

Received 20 May 2005 / Accepted 5 September 2005

ABSTRACT

We present VLT near-IR spectroscopic observations of three X-ray sources characterized by extremely high X-ray-to-optical ratios (X/O $>40)$, extremely red colors $(6.3<R-K<7.4$, i.e. EROs) and bright infrared magnitudes $(17.6<K<18.3)$. These objects are very faint in the optical, making their spectroscopic identification extremely challenging. Instead, our near-IR spectroscopic observations have been successful in identifying the redshift of two of them $(z=2.08$ and $z=1.35)$, and tentatively even of the third one $(z=2.13)$. When combined with the X-ray properties, our results clearly indicate that all these objects host obscured QSOs $\left(4 \times 10^{44}<L_{2-10 \mathrm{keV}}<1.5 \times 10^{45} \mathrm{erg} \mathrm{s}^{-1}\right.$, $2 \times 10^{22}<N_{\mathrm{H}}<4 \times 10^{23} \mathrm{~cm}^{-2}$ ) at high redshift. The only object with unresolved morphology in the $K$ band shows broad H $\alpha$ emission, but not broad $\mathrm{H} \beta$, implying a type 1.9 AGN classification. The other two objects are resolved and dominated by the host galaxy light in the $K$ band, and appear relatively quiescent: one of them has a LINER-like emission line spectrum and the other presents only a single, weak emission line which we tentatively identify with $\mathrm{H} \alpha$. The galaxy luminosities for the latter two objects are an order of magnitude brighter than typical local $L_{K}^{*}$ galaxies and the derived stellar masses are well in excess of $10^{11} M_{\odot}$. For these objects we estimate black hole masses higher than $10^{9} M_{\odot}$ and we infer that they are radiating at Eddington ratios $L / L_{\mathrm{Edd}} \leq 0.1$. We discuss the implications of these findings for the coevolution of galaxies and black hole growth. Our results provide further support that X-ray sources with high X/O ratios and very red colors tend to host obscured QSO in very massive galaxies at high redshift.

Key words. galaxies: active - quasars: emission lines - quasars: general - infrared: galaxies - X-rays: galaxies

\section{Introduction}

During the past few years hard X-ray surveys have clearly revealed the important role played by obscured AGNs for the cosmic X-ray background and for the accretion history of supermassive black holes (Brandt et al. 2001; Giacconi et al. 2002; Hasinger et al. 2001; Fiore et al. 2003; Ueda et al. 2003;

* Based on data obtained at the VLT through the ESO program 73.A-0598(A).
Marconi et al. 2004; Barger et al. 2005). Obscured AGNs are found to be 3-4 times more numerous than unobscured AGNs. This figure seems to decrease at higher, QSO-like luminosities (Fiore et al. 2003; Ueda et al. 2003; La Franca et al. 2005), although this trend has been recently questioned by Treister et al. (2005). One of the main issues affecting these studies (and in particular the obscured-to-unobscured QSO ratio) is that a significant fraction of high- $z$, luminous obscured AGNs may have escaped optical spectroscopic identification due to the 
weakness of their optical counterparts. In most of these cases multiband photometry is the only viable resource to constrain their redshift.

Within this context a new interesting class of objects, emerging from the X-ray surveys, are sources with very high $\mathrm{X}$-ray-to-optical ratio (hereafter $\mathrm{X} / \mathrm{O})^{1}$, and in particular those with $\mathrm{X} / \mathrm{O}>10$ (to be compared with the value of $\mathrm{X} / \mathrm{O} \sim 1$ of unobscured, type 1 AGNs). In deep surveys $\left(F_{2-10 \mathrm{keV}}<\right.$ $10^{-14} \mathrm{erg} \mathrm{cm}^{-2} \mathrm{~s}^{-1}$ ) very few sources with $\mathrm{X} / \mathrm{O}>10$ have been identified spectroscopically (Barger et al. 2003; Mainieri et al. 2005 ), since the combination of low X-ray fluxes and high X/O results into optical magnitudes $R>25$, extremely difficult to observe spectroscopically even with the largest telescopes currently available. Instead, large area shallower surveys, such as the HELLAS2XMM survey $\left(F_{2-10 \mathrm{keV}}>10^{-14} \mathrm{erg} \mathrm{cm}^{-2} \mathrm{~s}^{-1}\right.$ over 1 sq. degree), delivered samples of high-X/O sources with brighter optical counterparts, therefore suitable for spectroscopic follow-up. In particular, Fiore et al. (2003) have spectroscopically identified a sizable sample (13 sources) of HELLAS2XMM sources with X/O $>10$ and $R \sim 24$, many of which are type 2 QSOs at high redshift.

However, a subsample of these HELLAS2XMM sources with extreme $\mathrm{X} / \mathrm{O}$ ratios $(30<\mathrm{X} / \mathrm{O}<150)$ are difficult to identify spectroscopically even within the HELLAS2XMM sample, due to the extremely faint optical magnitudes of their counterparts. Mignoli et al. (2004) obtained near-IR, $K$-band images of 10 sources characterized by $\mathrm{X} / \mathrm{O}>30$ and $R>24.5$. The most surprising finding were the bright near-IR magnitudes of these objects, in the range $17.6<K_{\mathrm{S}}<19.1$, resulting into colors $R-K>5$, placing them into the class of Extremely Red Objects (EROs). Thanks to excellent seeing conditions, most of the sources were resolved, showing elliptical-like profiles in most cases (but two point-like sources and one disky-profile are also present). Colors and sizes suggest redshifts larger than about 1. When combined with the X-ray fluxes and slopes, their results suggest that these sources host type 2 QSOs, whose light is totally absorbed in the optical and often even in the near-IR. Such objects with extreme $\mathrm{X} / \mathrm{O}$ ratios and extremely red colors probably represent $10 \%$ of the sources with $L_{X}>10^{44} \mathrm{erg} \mathrm{s}^{-1}$ in the HELLAS2XMM sample (Mignoli et al. 2004). Obviously, a spectroscopic investigation and redshift confirmation of these sources is highly desirable, and would help us to tackle the following issues: 1) confirm the QSO2 nature of the "superEROs" in the Mignoli et al. (2004) sample; 2) determine their contribution to the census of obscured AGNs at high redshift and compare it with the models of the X-ray background (Perola et al. 2004; Fiore et al. 2003; Mainieri et al. 2005; La Franca et al. $2005)$; 3) investigate further the trends of the AGN2/AGN1 ratio as a function of luminosity and redshift (Fiore et al. 2003; Ueda et al. 2003; La Franca et al. 2005); 4) confirm that these $\mathrm{X}$-rays sources are hosted in very massive galaxies at high redshift, and investigate the implications for the QSO-spheroids coevolution.

\footnotetext{
${ }^{1} \mathrm{X} / \mathrm{O}=F_{\mathrm{X}} / F_{\mathrm{opt}}=F_{\mathrm{X}} / 10^{-(0.4 R+5.4)}$, where $F_{\mathrm{X}}$ is the observed $\mathrm{X}$-ray flux in the band $2-10 \mathrm{keV}$ (in units of $\mathrm{erg} \mathrm{s}^{-1} \mathrm{~cm}^{-2}$ ) and $R$ is the $R$-band magnitude.
}

Table 1. Summary of the targets properties.

\begin{tabular}{lcccl}
\hline \hline Source & $\mathrm{X} / \mathrm{O}$ & $K_{\mathrm{s}}$ & $R-K_{\mathrm{s}}$ & Morph. $(K)$ \\
\hline Abell2690\#029 & 78 & 17.68 & $7.4 \pm 1.0$ & Point. \\
BPM16274\#069 & 52 & 17.83 & $6.6 \pm 0.8$ & Ell./Disc. \\
Abel12690\#075 & 45 & 18.32 & $6.3 \pm 0.7$ & Ellip. \\
PKS0537-28\#111 & 154 & 17.66 & $6.8 \pm 0.7$ & Ellip. \\
\hline
\end{tabular}

The relatively bright magnitudes characterizing the near-IR counterparts of the X-ray sources in the Mignoli et al. (2004) sample suggest that near-IR spectroscopy may be a more effective tool to investigate these extremely red sources than optical spectroscopy. Moreover, the rest-frame optical AGN lines could emerge in the near-IR, since dust extinction is reduced with respect to the rest-frame UV lines. We have performed a pilot program of near-IR spectroscopy of four HELLAS2XMM sources with $R-K>6$, although we could obtain a full nearIR spectrum only for three of them. As discussed in this paper, the results are extremely encouraging, with the determination of secure spectroscopic redshifts for two sources and a tentative redshift for the third one.

In Sect. 2 we describe the sample selection, along with the observations and the data reduction process. In Sect. 3 we present the resulting spectra and discuss each source individually. In Sect. 4.1 we discuss the implications of our results for the connection between QSO2, EROs and high X/O sources. In Sect. 4.2 we infer the stellar masses of the host galaxies, the black hole masses and the accretion rates in terms of Eddington luminosity. Finally, in Sect. 5 we draw the main conclusions.

In this paper we assume the concordance $\Lambda$-cosmology with $H_{0}=71 \mathrm{~km} \mathrm{~s}^{-1} \mathrm{Mpc}^{-1}, \Omega_{\Lambda}=0.73$ and $\Omega_{\mathrm{m}}=0.27$ (Spergel et al. 2003).

\section{Sample selection, observations and data reduction}

Out of the $11 \mathrm{X}$-ray HELLAS2XMM high X/O sources in the Mignoli et al. (2004) sample (10 of which have EROs colors), we selected four sources with $6.3<R-K<7.4, K<18.3$, $\mathrm{X} / \mathrm{O}>40$ and different morphological types. Table 1 summarizes the properties of the sources in our pilot program. Figure 1 shows the location of our four sources in a $K$ vs. $R-K$ diagram (big squared dots). The figure highlights the extreme properties of our "super-EROs", in terms of colors and brightness, relative to the population of X-ray sources found in deep pencil surveys, such as the CDFN (Barger et al. 2003), whose distribution is indicated with small symbols ${ }^{2}$. Other shallow surveys have found X-ray sources with near-IR counterparts with similar extreme properies (Gandhi et al. 2004; Severgnini et al. 2005; Brusa et al. 2005). However, within this class of X-ray super-EROs the ones presented in this paper are among the few ones with rest-frame optical spectra which allow, beside the redshift determination, to investigate their optical properties.

\footnotetext{
${ }^{2}$ Barger et al. (2003) list the photometry in the $H K^{\prime}$ filter. We converted this photometry into $K$ band by using the relation $H K^{\prime}-K=$ $0.13+0.05(I-K)$, provided in the same paper.
} 


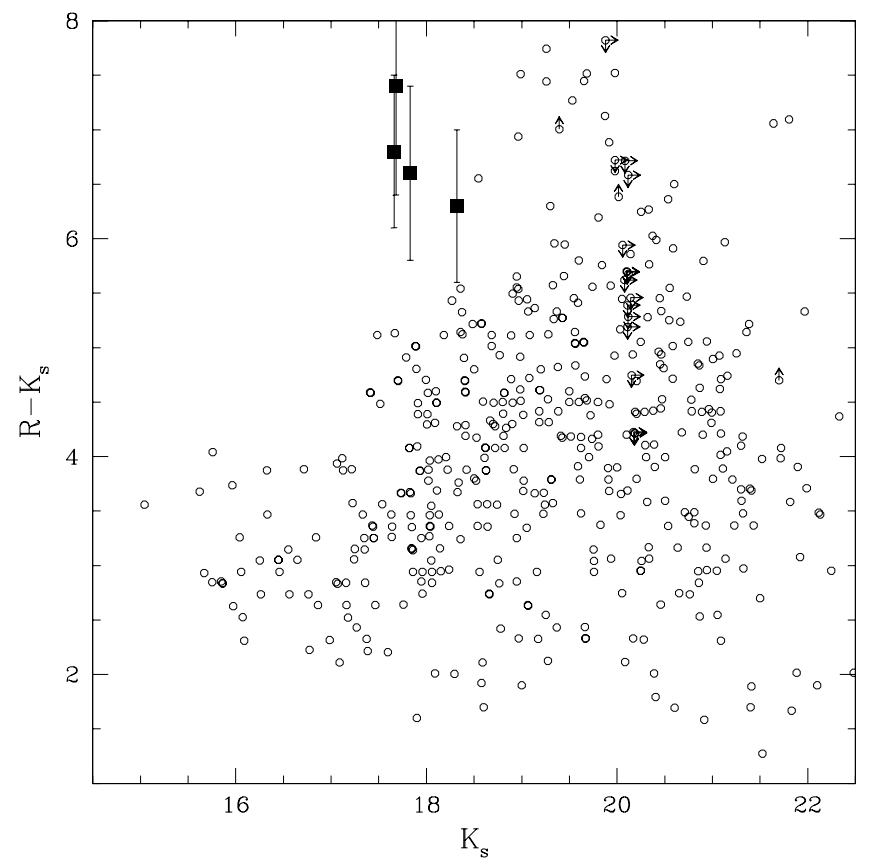

Fig. 1. Comparison of the $K$-band fluxes and $R-K$ colors of the sources in our sample (large solid symbols) with the $\mathrm{X}$-ray sources in the deep, pencil beam survey in the Chandra Deep Field North (small empty symbols Barger et al. 2003). Note the extreme properties of our objects in terms of brightness and colors which sample a region totally unexplored by the deep surveys.

Observations were obtained with ISAAC at ESO-VLT during period 73 (April 2005 - September 2005) in service mode. We used the low resolution mode with $1^{\prime \prime}$ slit $(R \sim 500)$ in the $J(1.16-1.34 \mu \mathrm{m}), H(1.48-1.76 \mu \mathrm{m})$ and $K(1.97-2.5 \mu \mathrm{m})$ bands. PKS0537\#111 is the only object which was observed only in the $H$ band. For each band we used eight integrations of $300 \mathrm{~s}$ each, and moving the target along the slit (following an A-B-B-A pattern), for a total of $40 \mathrm{~min}$ of integration per band. In some cases the observation was repeated due to the poor seeing in the first observation $\left(>1.2^{\prime \prime}\right)$.

Data reduction followed the standard threads. The background was removed by subtracting contiguous A-B frames. Flat fielding was performed by using the master flat provided by the ESO pipeline. Spectroscopic calibration was obtained through the spectrum of an arc (Ar-Xe) lamp. Small wavelength shifts due to the grating positioning uncertainty were corrected by means of the $\mathrm{OH}$ sky lines. The individual frames were aligned and averaged, with a sigma-clipping threshold to remove bad pixels and cosmic rays. Telluric absorption features and the relative response of the instrument were corrected by dividing the spectrum by a stellar standard of known spectral type $^{3}$. The absolute flux calibration (and therefore also interband calibration) is problematic. Indeed, we do not have photometry for the targets in the $J$ and $H$ bands which could be used to carefully calibrate the spectra. So we attempted to calibrate the spectra by using the standard star taken with the slit

\footnotetext{
3 The intrinsic shape of the standard stars was corrected by using the spectral libraries of Pickles (1998) or through the method discussed in Maiolino et al. (1996) in the case of late type main sequence stars.
}

of $2^{\prime \prime}$ and trying to estimate the slit losses through the seeing observed in the acquisition images. We checked that in the $K$ band (where we have photometry) the accuracy of the flux calibration is about $25 \%$, but we do not have any control for the $J$ and $H$ bands. Moreover, in the case of BPM16724\#069 no standard star was taken, so we had to rely on standard stars taken 10-20 nights earlier, implying larger uncertainties both for the absolute flux calibration and even for the intra-band slope and relative calibration.

\section{Results}

The single $H$-band spectrum of PKS0537\#111 is featureless. The limited spectral coverage did not allow us to provide useful constraints on the redshift or on the nature of the source, other than supporting the stellar nature of the near-IR light inferred from the $K$ band image by Mignoli et al. (2004). This source is no further discussed.

Figures 2-4 show the resulting spectra of the three sources observed in all the three bands, smoothed with a boxcar of 6 pixels (which corresponds to the projected slit width) to improve the $\mathrm{S} / \mathrm{N}$ without significantly affecting the spectral resolution (the final effective resolution after this smoothing is 420). Pixels were rebinned to half of the spectral resolution element. The main observational results are reported in Table 2 . We discuss each individual object in the following.

\subsection{Abell2690\#029}

The spectrum shows a red continuum with a prominent broad $\mathrm{H} \alpha\left(F W H M \sim 4200 \mathrm{~km} \mathrm{~s}^{-1}\right)$ in the $K$ band, whose identification is supported by the detection of [OIII]5007 $\AA$ in the $H$ band. The [SII] doublet at $6717+6731 \AA$ is also detected (any [NII]6584 $\AA$ line is blended with the broad H $\alpha$ ). These emission lines imply a redshift of 2.087 , close to the redshift of 2.4 inferred by Fiore et al. (2003), based on the X/O- $L_{X}$ correlation for obscured systems, and by Mignoli et al. (2004), based on the colors. Broad $\mathrm{H} \beta$ in the $H$ band is not detected, yielding to a "type 1.9" classification for the AGN (e.g. Osterbrock \& Martel 1993). Note that the narrow feature near to the expected location of $\mathrm{H} \beta$ is probably a bad subtraction of the very strong sky line at this wavelength, although some contribution from a narrow component of $\mathrm{H} \beta$ cannot be excluded.

From the lower limit on the $\mathrm{H} \alpha / \mathrm{H} \beta$ broad lines ratio we can set a lower limit to the extinction in the range $A_{\mathrm{V}}>2-5 \mathrm{mag}$, depending on the assumed intrinsic value of the Balmer ratio (see discussion in Maiolino et al. 2001a) and on the assumed extinction curve (Gaskell et al. 2004; Hopkins et al. 2004; Maiolino et al. 2001a). On the other hand, the detection of a broad $\mathrm{H} \alpha$ requires the visual extinction not to be much higher than $\sim 5 \mathrm{mag}$, otherwise the corrected $\mathrm{H} \alpha$ flux would imply a ratio $L(\mathrm{H} \alpha) / L_{\mathrm{X}} \gg 1$, i.e. much larger than observed even in the most extreme AGNs (Koratkar et al. 1995).

At a redshift of 2.087 the fit of the X-ray spectrum gives an absorbing column density of $2.2 \times 10^{22} \mathrm{~cm}^{-2}$ 


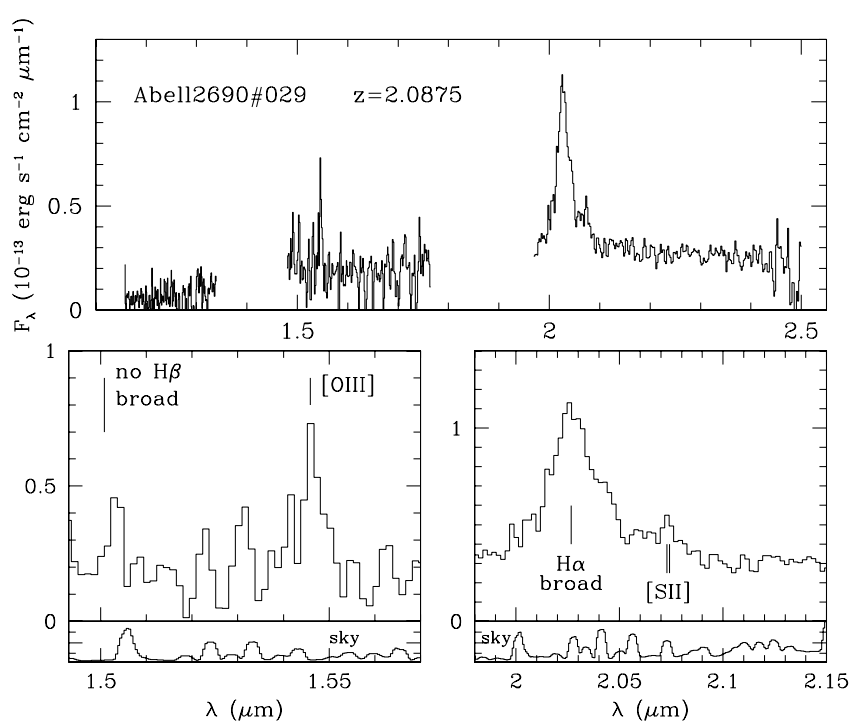

Fig. 2. ISAAC-VLT near infrared spectrum of Abell2690\#029, a type 1.9 , red QSO at $z=2.087$. The spectrum is smoothed with a 6 pixel boxcar. The bottom panels show a zoom of the spectrum around [OIII] and $\mathrm{H} \alpha$. The lower insets show the sky spectrum (arbitrary flux units).

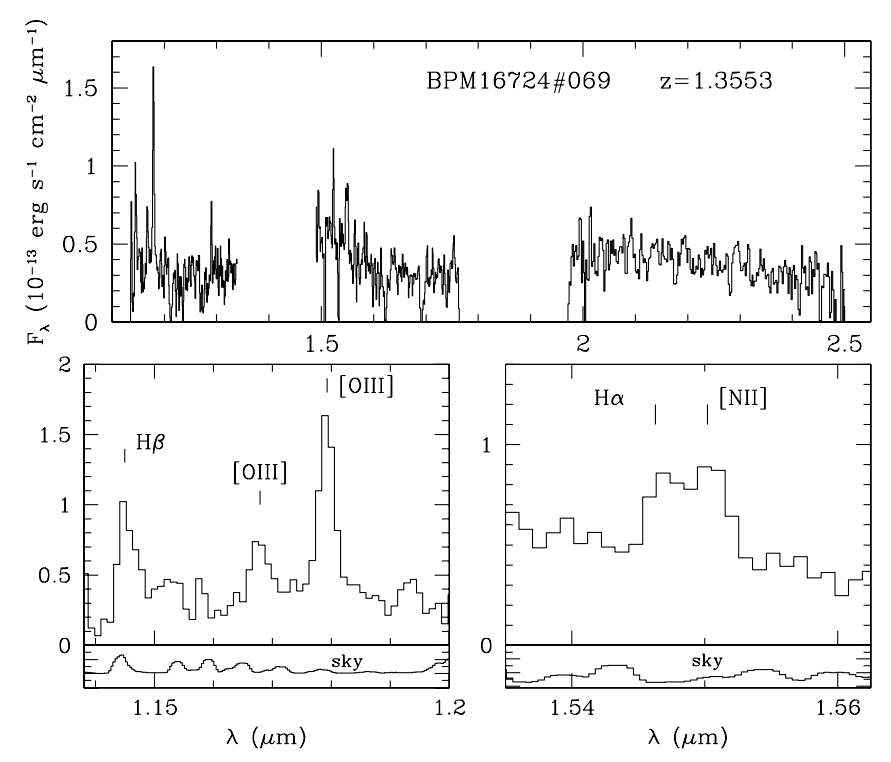

Fig. 3. As Fig. 2 for BPM16724\#069, a LINER-like AGN at $z=1.355$.

(Perola et al. 2004) ${ }^{4}$. The implied 2-10 keV luminosity corrected for absorption is $\sim 10^{45} \mathrm{erg} \mathrm{s}^{-1}$, placing this object in the class of obscured QSOs.

\footnotetext{
${ }^{4}$ The extinction expected from the X-ray absorbing column density assuming a Galactic gas-to-dust ratio and extinction curve is higher $\left(A_{\mathrm{V}}(\exp ) \sim 10 \mathrm{mag}\right)$ than inferred from the near-IR spectrum, but marginally consistent given the errors on $N_{\mathrm{H}}$. Moreover, a mismatch between optical and X-ray absorption is common to most AGNs (Maiolino et al. 2001b).
}

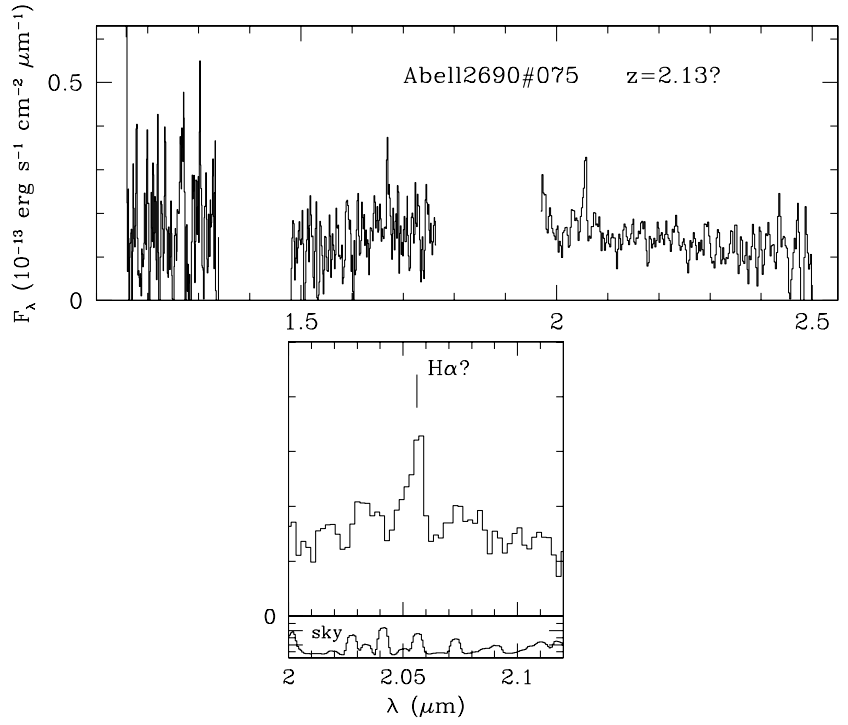

Fig. 4. As Fig. 2 for Abell2690\#075. The bottom panel shows the zoom around a line tentatively identified as $\mathrm{H} \alpha$ at $z=2.13$.

\section{2. $B P M 16274 \# 069$}

A redshift of 1.355 is secured by the detection of [OIII] $4960+5007 \AA$ and (narrow) $\mathrm{H} \beta$ in the $J$ band and a noisier detection of (narrow) $\mathrm{H} \alpha$ and [NII] 6584 in the $H$ band. Such a redshift is close to the values inferred both by Fiore et al. (2003) and Mignoli et al. (2004), 1.6 and >1.4 respectively, based on the X-ray, optical and near-IR photometric properties, as discussed above.

The line ratios $(\mathrm{H} \beta /[\mathrm{OIII}]=1.03,[\mathrm{NII}] / \mathrm{H} \alpha=1.0)$ are in the LINERs range, which is not unusual even in several obscured, intrinsically powerful nearby AGNs (Maiolino et al. 2003). The ratio of $\mathrm{H} \alpha / \mathrm{H} \beta \sim 1$ is unusually low, and probably reflects the large uncertainties affecting the inter-calibrations of the $J$ and $H$ bands of this object (Sect. 2).

Also for this object the spectroscopic redshift implies substantial gaseous column density $\left(\sim 2.2 \times 10^{22} \mathrm{~cm}^{-2}\right)$ along the line of sight and a high intrinsic luminosity (Table 2 ), yielding to a type 2 QSO classification.

\subsection{Abell2690\#075}

There is only one tentative line detection at $\sim 2.05 \mu \mathrm{m}$. If identified with $\mathrm{H} \alpha$ the inferred redshift would be 2.13 . This is very close to the redshift of 1.9 inferred by Fiore et al. (2003), based on the $\mathrm{X} / \mathrm{O}-L_{\mathrm{X}}$ correlation, and consistent with the lower limit of 1.30 inferred by Mignoli et al. (2004).

Alternatively, the line at $2.05 \mu \mathrm{m}$ could be [OIII]5007 $\AA$, implying a redshift of 3.11 . This could be corroborated by a very marginal detection of a feature at $1.59 \mu \mathrm{m}$, which could match the wavelength expected for [NeIII] $3869 \AA$ and which often accompains [OIII]. Any other possible identification of the $2.05 \mu \mathrm{m}$ feature with other emission lines weaker than $\mathrm{H} \alpha$ and [OIII] is unlikely.

Higher signal-to-noise spectra are required to better confirm the line(s) detection and the redshift. In this paper we will assume the working hypothesis that the redshift is 2.13 , i.e. 
Table 2. Main results from the observations.

\begin{tabular}{|c|c|c|c|c|c|c|c|}
\hline \multirow[b]{2}{*}{ Source } & \multirow[b]{2}{*}{$z$} & \multicolumn{4}{|c|}{ Emission lines } & \multirow[b]{2}{*}{$\begin{array}{c}N_{\mathrm{H}} \\
\left(10^{22} \mathrm{~cm}^{-2}\right)\end{array}$} & \multirow[b]{2}{*}{$\begin{array}{c}L_{2-10 \mathrm{keV}} \\
\left(10^{44} \mathrm{erg} \mathrm{s}^{-1}\right)\end{array}$} \\
\hline & & Id. & $\begin{array}{l}\lambda_{\mathrm{obs}} \\
(\mu \mathrm{m})\end{array}$ & $\begin{array}{c}\text { Flux } \\
10^{-16} \mathrm{erg} \mathrm{cm}^{-2} \mathrm{~s}^{-1}\end{array}$ & $\begin{array}{c}E W \text { (rest) } \\
\AA\end{array}$ & & \\
\hline \multirow[t]{3}{*}{ Abell2690\#029 } & 2.0875 & $\mathrm{H} \alpha$ (broad) & $2.0266 \pm 0.0015$ & $20 \pm 4$ & $260 \pm 50$ & $2.2_{-1.7}^{+2.7}$ & 9.7 \\
\hline & & [OIII] 5007 & $1.5457 \pm 0.0008$ & $1.2 \pm 0.2$ & $37 \pm 7$ & & \\
\hline & & $\mathrm{H} \beta$ (broad) & - & $<1$ & - & & \\
\hline \multirow[t]{5}{*}{ BPM16274\#069 } & 1.3553 & [OIII] $] 5007$ & $1.1790 \pm 0.0002$ & $4.6 \pm 0.5^{a}$ & $43 \pm 5$ & $2.6_{-10}^{+1.5}$ & 3.9 \\
\hline & & [OIII] 4960 & $1.1680 \pm 0.0005$ & $1.7 \pm 0.5^{a}$ & $13 \pm 4$ & & \\
\hline & & $\mathrm{H} \beta$ (narrow) & $1.1446 \pm 0.0005$ & $2.1 \pm 0.6^{a}$ & $21 \pm 6$ & & \\
\hline & & $\mathrm{H} \alpha$ (narrow) & $1.5463 \pm 0.0005$ & $2.3 \pm 0.7^{a}$ & $10 \pm 3$ & & \\
\hline & & {$[\mathrm{NII}] 6583$} & $1.5502 \pm 0.0005$ & $2.8 \pm 0.7^{a}$ & $12 \pm 3$ & & \\
\hline Abell2690\#075 & $2.13^{b}$ & $\mathrm{H} \alpha(?)^{b}$ & $2.0574 \pm 0.0020^{b}$ & $0.81 \pm 0.15^{b}$ & $30 \pm 6$ & $36_{-22}^{+47 b}$ & $15.0^{b}$ \\
\hline
\end{tabular}

${ }^{a}$ Highly uncertain flux calibration. ${ }^{b}$ Redshift and other derived quantities based on the tentative identification of the feature at $2.0574 \mu \mathrm{m}$ with $\mathrm{H} \alpha$.

assuming that the tentative line detection is $\mathrm{H} \alpha$. Should the line turn out to be [OIII]5007 $\AA$ from future, higher quality spectra, then our conclusions would be further reinforced: a higher redshift would imply even higher X-ray luminosities, higher column densities and higher galaxy light and masses than estimated in the following of this paper.

The tentative spectroscopic redshift of 2.13 implies substantial absorbing $N_{\mathrm{H}}$ along the line of sight and a high intrinsic luminosity (Table 2), implying a type 2 QSO classification also in this case. We note that the X-ray spectrum of this source is so flat that it could also be consistent with a reflection-dominated Compton thick QSO. In the latter case the intrinsic X-ray luminosity would exceed $10^{46} \mathrm{erg} \mathrm{s}^{-1}$. Such an extreme QSO, in terms of obscuration, would be similar to the one found by Norman et al. (2002) in the CDFS, although intrinsically much more luminous.

\section{Discussion}

\subsection{QSO2 in super-EROs}

As outlined in the previous section and in Table 2, the redshift estimated through our spectra imply QSO-like luminosities $\left(L_{2-10 \mathrm{keV}}>3 \times 10^{44} \mathrm{erg} \mathrm{s}^{-1}\right)$ and significant gas absorption along the line of sight $\left(N_{\mathrm{H}}>2 \times 10^{22} \mathrm{~cm}^{-2}\right)$, i.e. all three objects host type 2, obscured QSOs. This class of sources has been long sought in the past. Recent campaigns of optical spectroscopic identification of X-ray sources (Barger et al. 2003; Fiore et al. 2003; Szokoly et al. 2004) are providing more cases of QSO2, but they are still under numerous with respect to unobscured, type 1 QSOs.

From the (rest-frame) optical point of view, only Abell2690\#029 presents the classical emission line properties of (dust-) obscured AGN (a "classical" type 1.9 spectrum). The other two do not present the classical emission lines of type 2 AGNs, one having a LINER-like spectrum and the other one being nearly featureless. However, such spectral properties are sometimes observed even in local heavily obscured AGNs (Maiolino et al. 2003). Probably, in these kind of AGNs the obscuring medium surrounds completely the nuclear source ("buried" AGN), therefore preventing ionizing photons to escape and form of a Narrow Line Region. Other cases of high- $z$

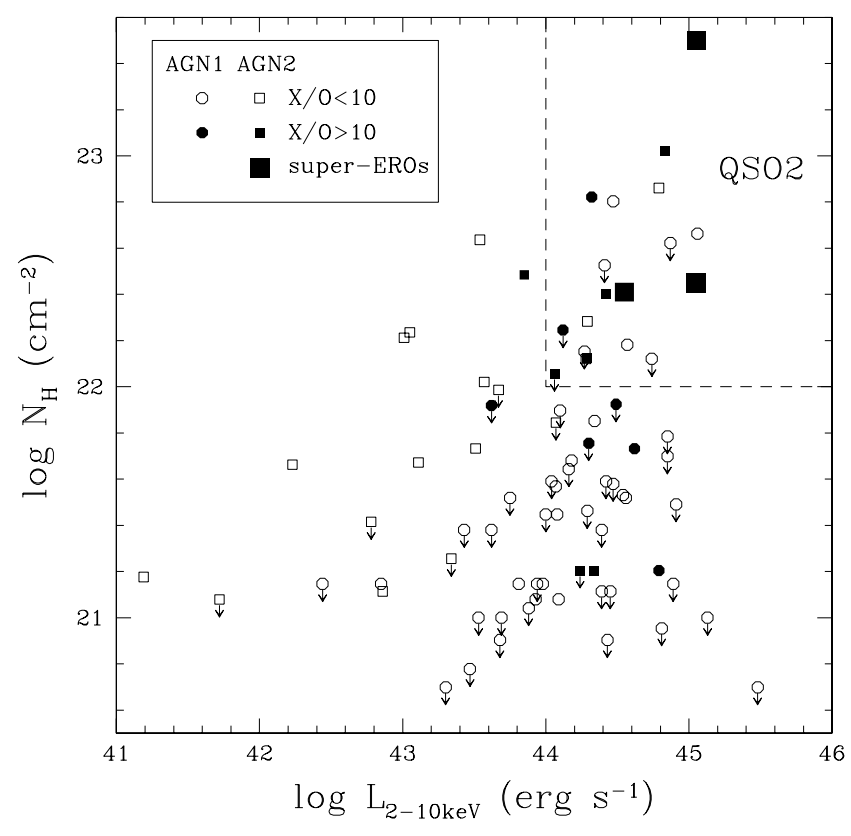

Fig. 5. $N_{\mathrm{H}}$ versus (absorption corrected) $L_{2-10 \mathrm{keV}}$ diagram where the sources identified through near-IR spectroscopy in this paper (large symbols) are compared with the other HELLAS2XMM sources identified through optical spectroscopy by Fiore et al. (2003) and whose X-ray spectra were analyzed by Perola et al. (2004). Circles and squares identify type 1 and type 2 AGNs according to the optical classification (see text for details). Solid and empty symbols indicate sources with $\mathrm{X} / \mathrm{O}>10$ and $\mathrm{X} / \mathrm{O}<10$, respectively. The region matching the definition of obscured, type 2 QSO is delimited by the dashed box.

X-ray sources with counterparts dominated by the host galaxy and with only one emission line $(\mathrm{H} \alpha)$ have been found by Severgnini et al. (2003), but in most of these cases the weakness of AGN signatures is ascribed to an intrinsic faintness of the AGN relative to the host galaxy.

Figure 5 shows a $N_{\mathrm{H}}-L_{\mathrm{X}}$ diagram, where we compare the three sources investigated in this paper (large symbols) with the HELLAS2XMM sources identified through optical spectroscopy by Fiore et al. (2003) and analyzed, for what concerns the X-ray spectral properties, by Perola et al. (2004) (small symbols). Circles and squares identify type 1 and type 2 AGN, 
respectively, according to the optical spectrum (here we include in the "type 2" class all those sources without broad emission lines, i.e. including "Emission Line Galaxies" and "Early Type Galaxies", see Fiore et al. 2003, for a detailed discussion). The dashed rectangle indicates the location of sources conventionally defined as obscured QSO (e.g. Mainieri et al. 2002). Although the works by Fiore et al. (2003) and Perola et al. (2004) have delivered a number of new QSO2, only 10\% of their whole spectroscopic sample can be safely identified as QSO2. Conversely, all three "super-EROs" identified is the current work are well within the region of QSO2.

In Fig. 5 we distinguish sources with high and low $\mathrm{X} / \mathrm{O}$ ratios $(\mathrm{X} / \mathrm{O}>10$ and $\mathrm{X} / \mathrm{O}<10)$ by marking them through solid and empty symbols (a similar plot, $N_{\mathrm{H}}$ vs. X/O, was shown by Comastri \& Fiore 2004). It is interesting to note that most sources with $\mathrm{X} / \mathrm{O}>10$ are characterized by $L_{\mathrm{X}}>10^{44} \mathrm{erg} \mathrm{s}^{-1}$ and half of them are absorbed (i.e. QSO2). On the opposite the low luminosity, Sy-like region is mostly populated by sources with $\mathrm{X} / \mathrm{O}<10$ and mostly unabsorbed. This result indicates that selecting sources with high $\mathrm{X} / \mathrm{O}$ is an efficient way to find obscured QSOs at high redshift, as already pointed out by Fiore et al. (2003). Our findings indicate that by combining this selection technique with the extremely red colors (EROs) the selection efficiency of QSO2 may improves even further, as already suggested by previous studies (Brusa et al. 2005; Severgnini et al. 2005). In particular, our results suggest that by selecting extreme values of $\mathrm{X} / \mathrm{O}(>40)$ and extreme values of $R-K(>6)$ the QSO2 selection efficiency may approach $100 \%$.

The physical interpretation of our results is relatively straightforward. On the one hand the high X/O tends to select obscured AGNs, since obscuration affects preferentially the optical with respect to the hard X-rays. The redshift further moves this selection in favor of obscured systems, both because the observed optical emission samples the UV rest-frame radiation and because the observed $\mathrm{X}$-ray radiation samples the harder emission. On the other hand the very red colors favor the selection of high- $z$ objects, especially when the AGN emission is completely absorbed and the optical/near-IR radiation is therefore dominated by the host galaxy. At least at our X-ray flux limits high redshifts translate also into high X-ray (intrinsic) luminosities.

We note that our results are generally consistent with previous near-IR spectroscopic studies of optically faint sources, both in terms of redshift range and in terms of source characteristics: most of them are identified as obscured AGNs at high redshift (Gandhi et al. 2002; Willott et al. 2003, 2004; Severgnini et al. 2005). Altogether, our and previous works indicate that near-IR spectroscopy is a powerful tool to identify the redshift and the nature of optically faint sources.

\subsection{Very massive galaxies traced by super-EROs}

Two of the sources spectroscopically identified by us, BPM16274\#069 and Abell2690\#075, are dominated by the host galaxy light in the $K$ band (Table 1). Thanks to the spectroscopic redshift we can determine the stellar luminosity the host galaxies, as listed in Table 3. The inferred luminosities
Table 3. Rest-frame properties inferred for the two sources dominated by stellar light.

\begin{tabular}{lcccc}
\hline \hline Source & $\begin{array}{c}L_{K} \\
\left(L_{\odot}\right)\end{array}$ & $\begin{array}{c}M_{\text {star }} \\
\left(M_{\odot}\right)\end{array}$ & $\begin{array}{c}M_{\mathrm{BH}}^{\mathrm{a}} \\
\left(M_{\odot}\right)\end{array}$ & $L / L_{\mathrm{Edd}}^{\mathrm{b}}$ \\
\hline BPM16274\#069 & $8.3 \times 10^{11}$ & $4 \times 10^{11}$ & $2 \times 10^{9}$ & 0.04 \\
Abel12690\#075 & $1.7 \times 10^{12}$ & $8 \times 10^{11}$ & $3 \times 10^{9}$ & 0.10 \\
\hline
\end{tabular}

Notes: ${ }^{a}$ Inferred black hole mass assuming the $L_{\mathrm{K}}-M_{\mathrm{BH}}$ relation obtained by Marconi \& Hunt (2003) for local galaxies. ${ }^{b}$ Eddington ratio assuming a bolometric correction $L_{\mathrm{bol}} / L_{\mathrm{X}}=30$.

are $L_{K} \sim 10^{12} L_{\odot}$, i.e. about one order of magnitude higher than local $L_{K}^{*}$. The inferred stellar masses are well in excess of $10^{11} M_{\odot}$ in both cases. A similar result was obtained by Severgnini et al. (2005) through the near-IR spectroscopic identification of an X-ray ERO source with high X/O and bright in the $K$ band, i.e. with properties very similar to our sources. These results indicate that the near-IR spectroscopic identification of EROs X-ray sources with high $\mathrm{X} / \mathrm{O}$ is an efficient method also to find very massive galaxies at high redshifts, and therefore to investigate and observationally test the scenarios of co-evolution between massive spheroids and black hole accretion (Granato et al. 2004; Di Matteo et al. 2005).

If the relation between near-IR bulge stellar light and Black Hole mass (Marconi \& Hunt 2003) holds also at high redshift, then our result would imply that these two sources host black holes with masses of $2-3 \times 10^{9} M_{\odot}$. If compared with the $\mathrm{X}$-ray luminosity, and assuming a bolometric correction factor $L_{\text {bol }} / L_{\mathrm{X}}=30$, such a result would imply that these obscured QSOs are radiating at a fraction of about $0.04-0.10$ of their Eddington luminosity. These values are significantly lower than the average $L / L_{\text {Edd }} \sim 0.5$ inferred by Marconi et al. (2004) from the comparison of the high- $z$ X-ray luminosity functions with the local Black Hole relic mass density. Although the uncertainties are large, our results would suggest that these very massive Black Holes have already passed their rapidly accreting phase and are reaching their final masses with lower accretion rates. This is also a trend which is expected to happen at $z \sim 1-2$ for very massive Black Holes according to the analysis of Marconi et al. (2004), as illustrated in Fig. 8 of that paper. Our results are also consistent with the decresing $L / L_{\text {Edd }}$ at low redshift obtained by McLure \& Dunlop (2004).

Finally, the finding of very massive Black Holes, hosted within very massive (quiescent) galaxies and with low accretion rate is also consistent with the feedback models for the coevolution of QSO and spheroids Granato et al. (2004). Indeed such models expect that in the QSO phase most of the star formation and black hole growth has already occurred, while further accretion is quenced by the feedback from the QSO itself.

Similar results on $L / L_{\text {Edd }}$ were obtained by Comastri \& Fiore (2004) and by Brusa et al. (2005) on a sample of spectroscopically identified EROs, although less massive than the sample investigated in this paper.

\section{Conclusions}

We have reported the results of a pilot program of near-IR spectroscopy aimed at identifying four X-ray sources characterized 
by extremely high $\mathrm{X}$-ray-to-optical ratios $(\mathrm{X} / \mathrm{O}>40)$, extremely red colors $(6.3<R-K<7.4)$ and relatively bright infrared magnitudes $(17.6<K<18.3)$. The optical spectroscopic identification of these sources is very difficult due to the extremely faint or undetected optical counterparts. On the contrary, the near-IR spectroscopic identification results to be a relatively successful technique, allowing us to secure the redshift and spectroscopic classification of at least two sources, and possibly even a third one, even with modest integrations times at VLT (40 min per band). The only source for which spectroscopic constrains cannot be inferred is the one which was observed in one single band $(H)$.

One object has a red, type 1.9 AGN spectrum (broad $\mathrm{H} \alpha$, but not broad $\mathrm{H} \beta$ ), at a redshift $z=2.03$. The second one has a LINER-like emission spectrum, at a redshift $z=1.36$. The third one has only a faint emission line, which we tentatively identify with $\mathrm{H} \alpha$ at $z=2.13$. At these redshifts the shape of the $\mathrm{X}$-ray spectrum indicates the presence of gas absorption along the line of sight with column densities of $2 \times 10^{22}<N_{\mathrm{H}}<$ $4 \times 10^{23} \mathrm{~cm}^{-2}$. The spectroscopic redshifts also imply intrinsic $\mathrm{X}$-ray luminosities in the range $4 \times 10^{44}<L_{\mathrm{X}}<1.5 \times$ $10^{45} \mathrm{erg} \mathrm{s}^{-1}$, i.e. in the QSO luminosity range.

Our results corroborate previous studies suggesting that a selection criterion based on the high $\mathrm{X} / \mathrm{O}$ and red $R-K$ colors provide an efficent way to select absorbed QSOs at high redshift. Our study further suggests that by pushing these criteria to extreme values $(\mathrm{X} / \mathrm{O}>40$ and $R-K>6)$ the selection efficiency of QSO2s is probably close to $100 \%$.

Two of the sources for which we could determine the spectroscopic redshift are dominated by the host galaxy light in the $K$ band. The inferred stellar light is about $10^{12} L_{\odot}$, about an order of magnitude higher than local $L_{K}^{*}$ galaxies. The inferred stellar masses are well in excess of $10^{11} M_{\odot}$. These obscured QSOs are therefore hosted in quiescent, very massive galaxies, already fully assembled even at these high redshifts.

By exploiting the relation between stellar light and black hole mass we infer that these galaxies host black holes with masses higher than $10^{9} M_{\odot}$ and that the QSOs radiate at less than $10 \%$ of the Eddington luminosity. This result suggests that these very massive black holes have already passed their rapidly accreting phase and are reaching their final masses with lower accretion rates. The finding of very massive black holes, hosted within very massive (quiescent) galaxies and with low accretion rates is consistent with the feedback models for the co-evolution of QSO and spheroids.

Summarizing, our results suggest that the near-IR spectroscopic identification of X-ray sources with EROs colors and high $\mathrm{X} / \mathrm{O}$ is a promising method to find absorbed QSOs and very massive galaxies at high redshift, and therefore to investigate the coevolution of spheroids and black hole growth.
Acknowledgements. We thank the anonymous referee for useful comments. P.S. acknowledges a research fellowship from the National Institute for Astrophysics (INAF). This work was partially supported by the Italian Ministry for Univerisity (MIUR) through grant Cofin03-02-23 e and by INAF through grant PRIN/INAF/2003/270.

\section{References}

Barger, A. J., Cowie, L. L., Capak, P., et al. 2003, AJ, 126, 632

Barger, A. J., Cowie, L. L., Mushotzky, R. F., et al. 2005, AJ, 129, 578

Brandt, W. N., Alexander, D. M., Hornschemeier, A. E., et al. 2001, AJ, 122, 2810

Brusa, M., Comastri, A., Daddi, E., et al. 2005, A\&A, 432, 69

Comastri, A., \& Fiore, F. 2004 in Baryons on Cosmic Structures, Ap\&SS, 294, 63

Di Matteo, T., Springel, V., \& Hernquist, L. 2005, Nature, 433, 604

Fiore, F., Brusa, M., Cocchia, F., et al. 2003, A\&A, 409, 79

Gandhi, P., Crawford, C. S., \& Fabian, A. C. 2002, MNRAS, 337, 781

Gandhi, P., Crawford, C. S., Fabian, A. C., \& Johnstone, R. M. 2004, MNRAS, 348, 529

Gaskell, C. M., Goosmann, R. W., Antonucci, R. R. J., \& Whysong, D. H. 2004, ApJ, 616, 147

Giacconi, R., Zirm, A., Wang, J.-X., et al. 2002, ApJS, 139, 369

Granato, G. L., De Zotti, G., Silva, L., Bressan, A., \& Danese, L. 2004, ApJ, 600, 580

Hasinger, G., Altieri, B., Arnaud, M., et al. 2001, A\&A, 365, L45

Hopkins, P. F., Strauss, M. A., Hall, P. B., et al. 2004, AJ, 128, 1112

Koratkar, A., Deustua, S. E., Heckman, T., et al. 1995, ApJ, 440, 132

La Franca, F., et al. 2005, ApJ, submitted

Mainieri, V., Bergeron, J., Hasinger, G., et al. 2002, A\&A, 393, 425

Mainieri, V., Rosati, P., Tozzi, P., et al. 2005, A\&A, 437, 805

Maiolino, R., Rieke, G. H., \& Rieke, M. J. 1996, AJ, 111, 537

Maiolino, R., Marconi, A., \& Oliva, E. 2001a, A\&A, 365, 37

Maiolino, R., Marconi, A., Salvati, M., et al. 2001b, A\&A, 365, 28

Maiolino, R., Comastri, A., Gilli, R., et al. 2003, MNRAS, 344, L59

Marconi, A., \& Hunt, L. K. 2003, ApJ, 589, L21

Marconi, A., Risaliti, G., Gilli, R., et al. 2004, MNRAS, 351, 169

McLure, R. J., \& Dunlop, J. S. 2004, MNRAS, 352, 1390

Mignoli, M., Pozzetti, L., Comastri, A., et al. 2004, A\&A, 418, 827

Norman, C., Hasinger, G., Giacconi, R., et al. 2002, ApJ, 571, 218

Osterbrock, D. E., \& Martel, A. 1993, ApJ, 414, 552

Perola, G. C., Puccetti, S., Fiore, F., et al. 2004, A\&A, 421, 491

Pickles, A. J. 1998, PASP, 110, 863

Rigby, J. R., Rieke, G. H., Pérez-González, P. G., et al. 2005, ApJ, 627,134

Severgnini, P., Caccianiga, A., Braito, V., et al. 2003, A\&A, 406, 483 Severgnini, P., Della Ceca, R., Braito, V., et al. 2005, A\&A, 431, 87 Spergel, D. N., Verde, L., Peiris, H. V., et al. 2003, ApJS, 148, 175

Szokoly, G. P., Bergeron, J., Hasinger, G., et al. 2004, ApJS, 155, 271

Treister, E., Castander, F. J., Maccarone, T. J., et al. 2005, ApJ, 621, 104

Ueda, Y., Akiyama, M., Ohta, K., \& Miyaji, T. 2003, ApJ, 598, 886

Willott, C. J., Simpson, C., Almaini, O., et al. 2003, MNRAS, 339, 397

Willott, C. J., Simpson, C., Almaini, O., et al. 2004, ApJ, 610, 140 